\title{
Expansion of BCL2+ lymphocytes in cutaneous graft-versus host disease is associated with steroid resistance and poor prognosis
}

Johanna Strobl' ${ }^{1}$, Ram Vinay Pandey ${ }^{1}$, Thomas Krausgruber ${ }^{2}$, Fiona Tinnefeld ${ }^{1}$, Lisa Kleißl${ }^{1}$,

Merima Herac ${ }^{3}$, Werner Rabitsch ${ }^{4}$, Christoph Bock ${ }^{2,5}$, Georg Hopfinger ${ }^{4}$, Georg Stary ${ }^{1,2,6}$

1 Department of Dermatology, Medical University of Vienna, Vienna, Austria, 2 Clinical Institute for Pathology, Medical University of Vienna, Vienna, Austria, 3 Department of Internal Medicine I, Bone Marrow Transplantation, Medical University of Vienna, Vienna, Austria,

4 Ludwig Boltzmann Institute for Rare and Undiagnosed Diseases, 5 Center for Molecular Medicine (CeMM), Vienna, Austria

\section{Introduction}

Graft-versus-host disease (GVHD) remains a major cause of mortality after allogeneic hematopoietic stem cell transplantation (HSCT) and response to first-line therapy with glucocorticoids is often limited. To identify novel therapeutic targets for treatment and/or prophylaxis of GVHD, we performed RNA sequencing and Differentially Expressed Gene (DEG) analysis on sequentially isolated T cells of HSCT recipients (Figure 1). Furthermore, we analyzed blood, skin, gastrointestinal, liver and lung samples of patients with previously untreated acute or chronic GVHD by qPCR, flow cytometry and tissue immunofluorescence (Figures 2-4).

\section{Results}

In recipients who later developed GVHD, we observed early up-regulation of the anti-apoptotic molecule BCL2, which is targeted in chronic lymphocytic leukemia with a recently approved small molecule inhibitor. Furthermore, gastrointestinal tract, liver and skin affected by acute and/or chronic GVHD showed higher BCL2 mRNA expression compared to matched control groups. BCL2 protein levels were elevated in overall leukocytes and pathogenic cell subsets including CD8+ T lymphocytes in peripheral blood and skin of GVHD patients. Notably, high Bcl-2 expression levels correlated to steroid-refractory GVHD and increased transplantrelated mortality. In vitro inhibition of BCL2 in allo-reactions led to dosedependent apoptosis of $T$ cells and increase of CD4/CD8 ratio.

Distinct transcriptional profiles of $T$ cells from patients with and without GVHD after HSCT.

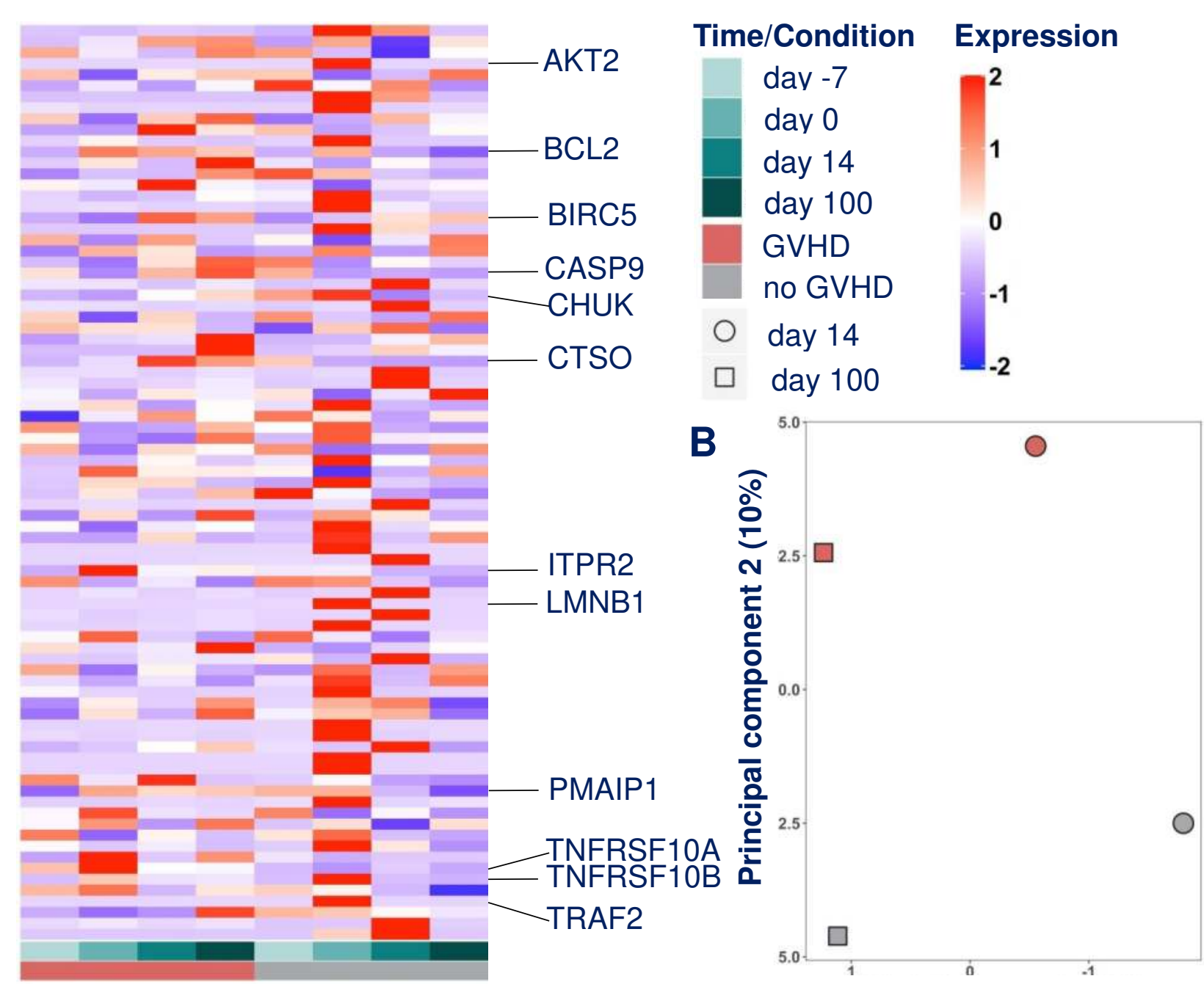

Principal component 1 (88\%)

Figure 1. A Heat map of 83 genes expressed with a $>1$ fold difference in mean in GVHD vs. no GVHD T cells. B Principal component analysis of T cells from GVHD and no GVHD patients at days 14 and 100 after HSCT.

$\mathrm{Bcl}-2$ is elevated in peripheral blood T cells of GVHD patients.
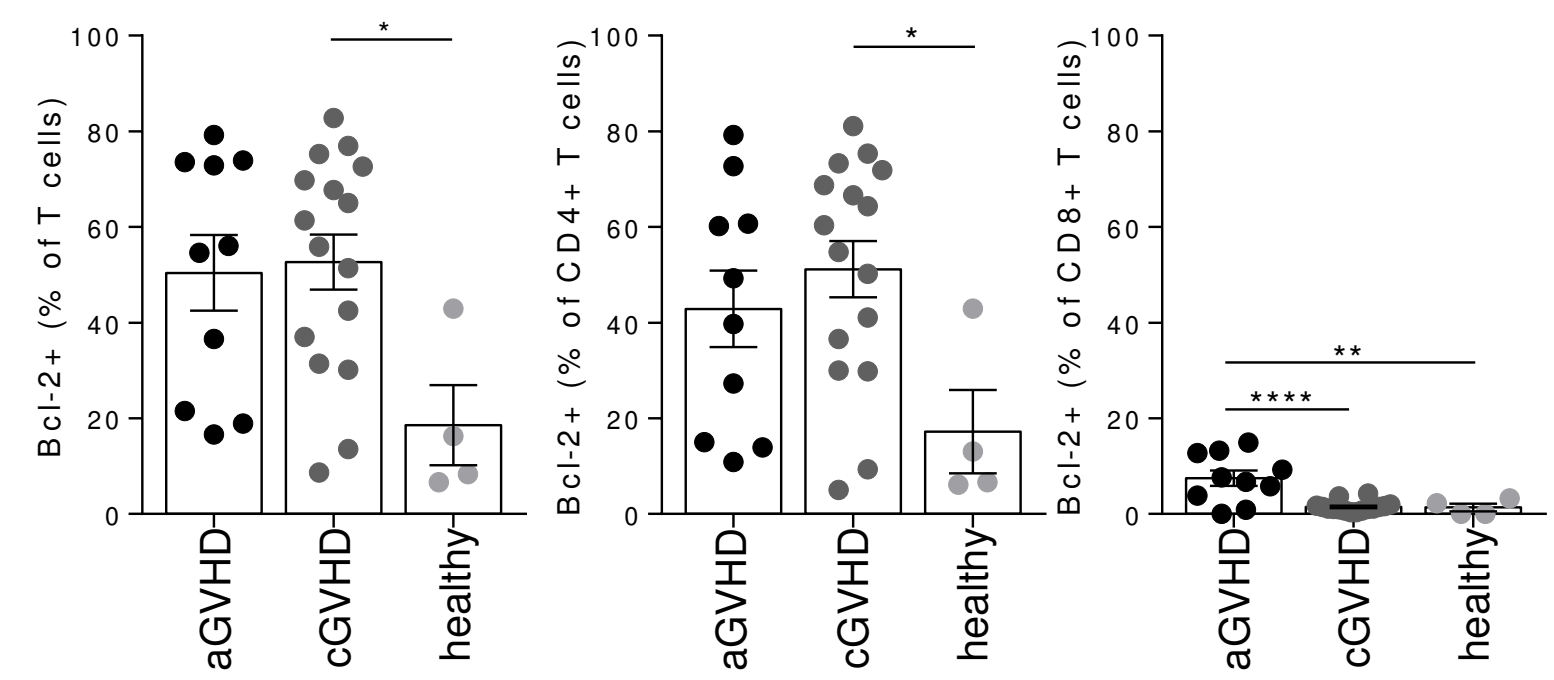

Figure 2. Relative numbers of Bcl-2 positive cells among PB T cells. Mean $\pm \mathrm{SD} ;{ }^{*} \mathrm{P}<0.05,{ }^{* *} \mathrm{P}<0.01,{ }^{* * *} \mathrm{P}<0.001$;
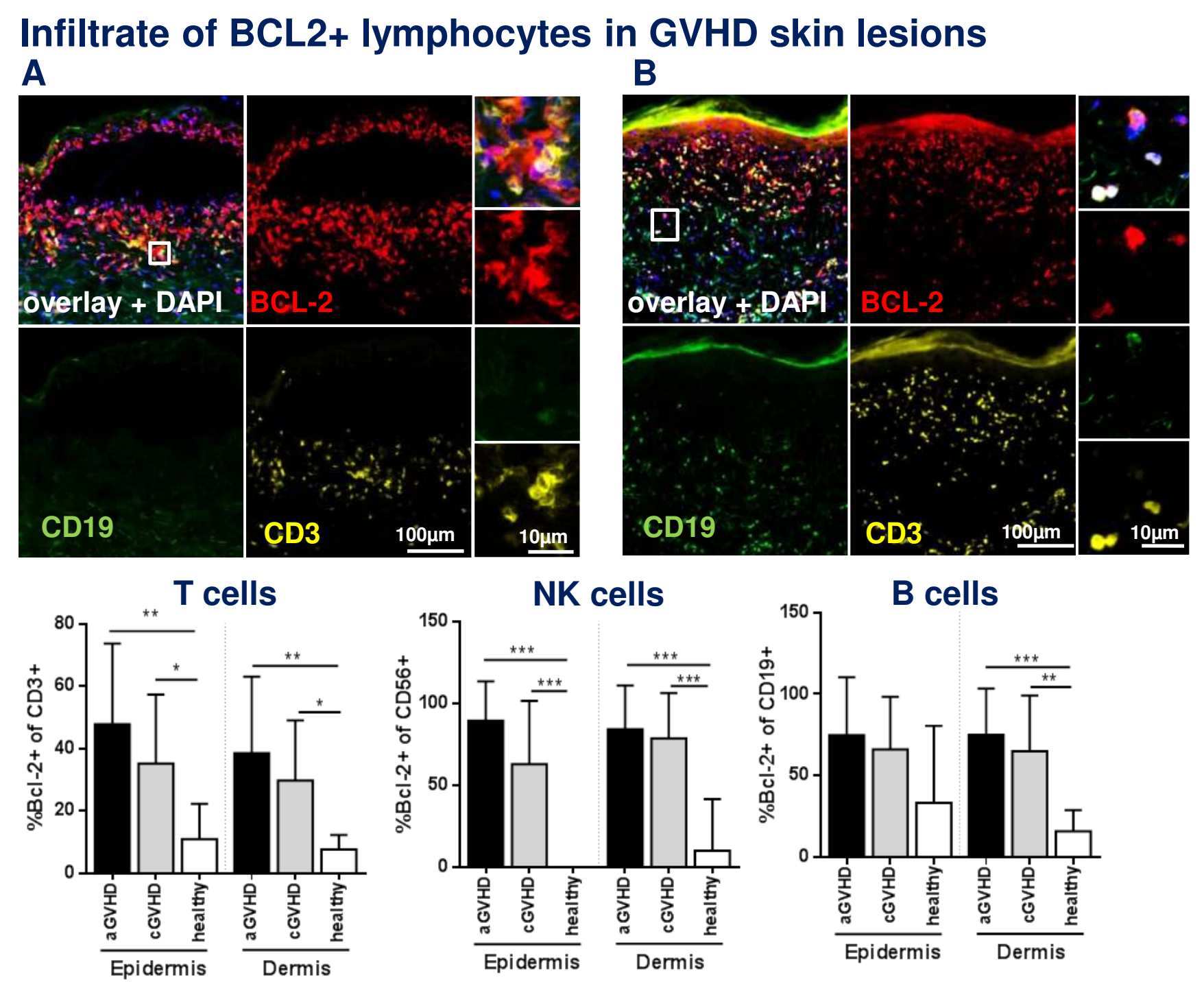

Figure 3. (A-B) Representative immunostaining of $\mathrm{Bcl}-2, \mathrm{~B}$ and $\mathrm{T}$ cells in aGVHD (A) and cGVHD (B). (C) Percentages of Bcl-2-expressing leukocytes in cutaneous GVHD. Mean $\pm S D$; ${ }^{*} P<0.05$, ${ }^{* *} P<0.01,{ }^{* * *} P<0.001$;

Elevated BCL2 mRNA in GVHD-tissues is associated with poor prognosis.

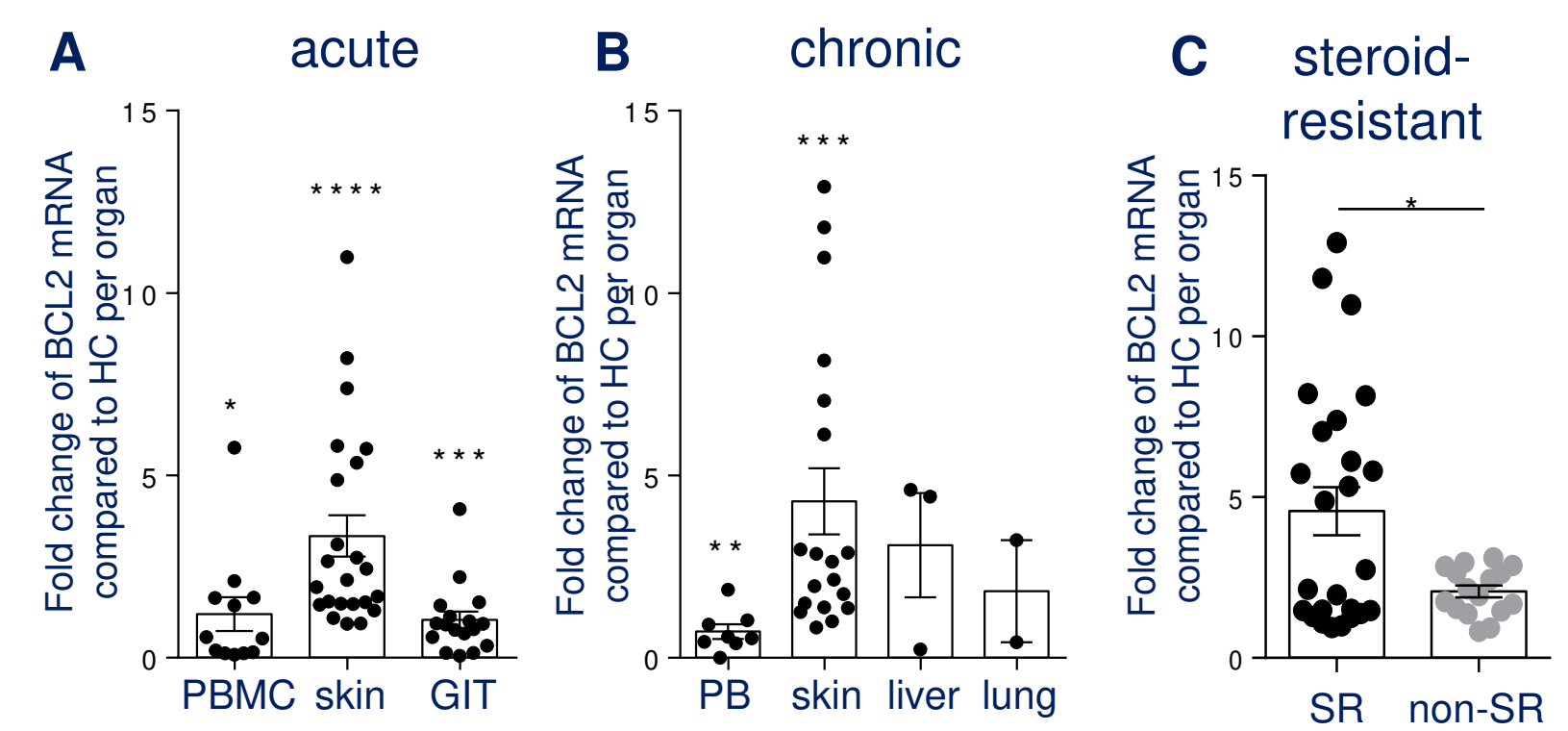

Figure 4. BCL2 expression in acute (A) and chronic (B) GVHD. C Comparison of steroid-resistant (SR) and non-SR cutaneous GVHD. Data shown as mean fold change over healthy control tissue \pm SEM; PB, peripheral blood.

BCL2 inhibition reduces allo-reactive steroid-resistant inflammatory $\mathrm{T}$ cells in vitro

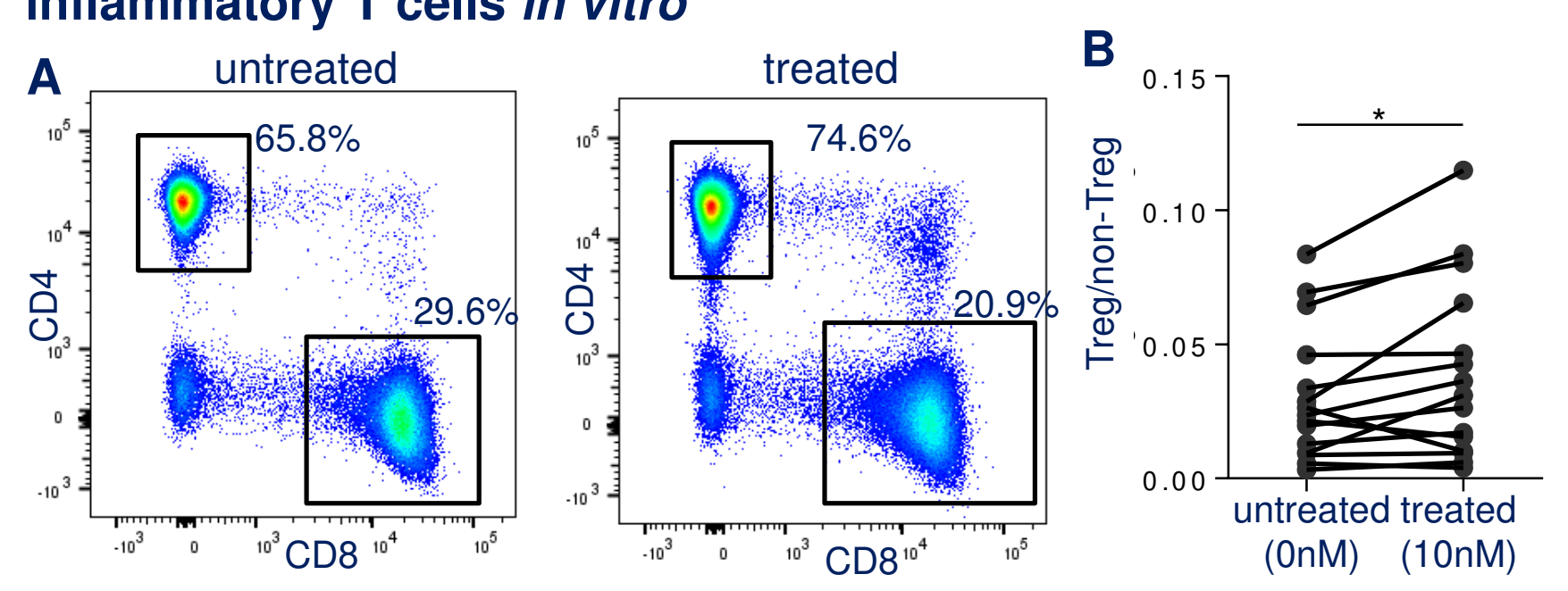

Figure 5. Selective apoptosis of $C D 8+T$ cells $(A)$ and non-regulatory $T$ cells (B) upon inhibition of BCL2 in PB isolated from SR-GVHD patients.

\section{Conclusion}

Our results highlight the role of $\mathrm{BCL2}$ as survival factor of GVHD-mediating lymphocytes. Selective inhibition of BCL2 may present a novel and urgently needed targeted therapy in treatment of steroid-refractory GVHD. 